\title{
BARS AS TRIGGERS OF GAS INFLOW
}

\author{
E. ATHANASSOULA \\ Observatoire de Marseille \\ 2, Place Le Verrier \\ 13248 Marseille Cedex 04, France
}

\begin{abstract}
I briefly discuss the flow of gas in and around bars and the amount of inflow it entails. I then focus on two ways of bringing gas very near the center of a barred galaxy. One relies on the absence of an inner Lindblad resonance and the other on the existence of bars within bars.
\end{abstract}

\section{Inflow in barred galaxy models}

In an axisymmetric unperturbed galaxy gas flows in circular flow lines around the center. In barred galaxies, however, gas flow has a complicated structure comprising both inflow and outflow, depending on the region considered. This has been recently addressed by Athanassoula (1992, hereafter A92), using a non-selfconsistent hydrodynamics code, initially written by G. D. van Albada (Van Albada, et al. 1982). If there are no shocks, then the flowlines are quasi-elliptical and the small net inflow is due to viscosity. On the other hand, if there are shocks, then, after hitting the shock, gas moves inwards at a high speed, with velocities often exceeding $100 \mathrm{~km} / \mathrm{sec}$. However this value is a local maximum and should not be confused with the average inflow velocity in a barred galaxy, which is considerably smaller. Indeed density weighted averages along a circle give values of only a few $\mathrm{km} / \mathrm{sec}$. The reason is that before the shock gas has a low density and moves outwards while at the shock it gets compressed and moves inwards with a high velocity. These effects can be seen in Figs. 2 to 10 of A92, or Fig. 1 of Athanassoula (1994, hereafter A94). The mean inflow velocity depends on the potential, on the radius where we do the averaging, and also on the way we model the gas flow.

An alternative way to measure inflow is to calculate the amount of mass 547

I. Appenzeller (ed.), Highlights of Astronomy, Vol. 10, 547-550.

(c) 1995 IAU. Printed in the Netherlands. 
within a given radius as a function of time, and then take the time derivative to find the inflow. This was done in A94 for a series of bar models and parameters describing the gas flow and for two different ways for describing the interstellar medium. In all cases the inflow had two distinct regimes. The first one corresponds to high inflow and is linked to the bar formation stage. The second one has a considerably smaller inflow and corresponds to the quasi-steady-state regime. A94, using the same code as in A92, showed that thinner bars with smaller pattern speeds and in more centrally concentrated potentials will produce more inflow. Also the inflow is higher if the viscosity of the code and/or the amount of recycling is larger.

To see whether these results are code dependent, the same calculations were repeated with a sticky particle code and collision laws following the precepts of Schwarz $(1981,1984)$. In this case the dust lanes are never as smooth and well defined as those observed, and it is not always easy to accomplish a steady state inflow regime for relatively long times. Nevertheless it is possible to find back, albeit much less clearly, the trends given by the hydro code. It would be interesting to measure the inflow for the same bar models now using SPH (Gingold and Monaghan 1977, Lucy 1977) to model the interstellar medium. Such a study is underway, but the results are not presently available for comparison. The cases available in the literature (e.g. Wada and Habe 1992; Friedli and Benz 1993, hereafter FB) show that for this model also there are two regimes, one of large and the second of smaller inflow, corresponding to the growth of the bar and its quasi-steady-state evolution. Furthermore figures $12 \mathrm{~d}$ and e of FB show that, in this case also, the inflow is higher for thinner, slower rotating bars, in good agreement with the results of the hydro response simulations (A94), particularly if one neglects from the FB figures model $\mathrm{E}$, which has a double bar system (Friedli, priv. com.).

\section{How far in can gas be pushed?}

\subsection{THE EXISTENCE OF AN INNER LINDBLAD RESONANCE}

As discussed in A94, in galaxies with no inner Lindblad resonance (hereafter ILR) it is possible to push gas much further inwards than in cases where an ILR is present. Thus the question arises whether barred galaxies have ILRs or not.

The inflow induced by the bar will enhance central concentration and therefore help the formation of an ILR (A92). Numerical simulations, however, show that the pattern speed of the galaxy also changes with time. In purely stellar simulations the pattern speed decreases with time (e.g. Little and Carlberg 1991). On the other hand simulations with both star and gas have a more complex behaviour (FB). During the initial phases 
the pattern speed decreases, but, as soon as a significant amount of gas has accumulated in the central regions, this trend is reversed and the pattern speed starts increasing very slowly. It will be interesting to see whether this behaviour is altered when gas recycling is introduced in these simulations.

A94 presented both theoretical and observational arguments that an ILR exists in a fair fraction of galaxies. This conclusion is strengthened by new observations of galaxies like NGC 1672 and NGC 1530 with the Maryland-Caltech Fabry-Perot system, showing evidence for ILRs near the edge of the bulge (Teuben et al., in preparation).

\subsection{BARS WITHIN BARS}

According to this scenario, due initially to Shlosman et al. (1989), as the bar pushes gas inwards it creates an unstable inner gas disc which then forms a secondary bar. This bar will in turn push gas inwards and a succession of such events can occur, each bringing the gas nearer to the center of the galaxy. Numerical simulations (Friedli and Martinet 1993, hereafter FM; Heller and Shlosman 1994; Combes 1994) confirmed this possibility, at least as far as the first secondary bar is concerned, while CO observations (e.g. Devereux et al. 1992) have found gaseous inner bars in a number of galaxies. Secondary bars have also been found in optical and infrared wavelengths (De Vaucouleurs 1974, 1975, FM, Shaw et al. 1993). Such secondary bars, of which a typical example is NGC 1291, are neither gaseous nor composed of predominantly young stars, so one needs more ingredients to the previous scenario. Two possibilities have so far been discussed in the literature.

a) The inner gas bar can force the stellar component into forming a bar (Shaw et al., 1993), in which case the pattern speed of the inner bar should be the same as that of the outer one.

b) The central stellar disc can also develop an instability. To make sure of that, FM introduced from the onset in some of their simulations an inner unstable disc. Alternatively one can rely on nonlinear mode-mode interactions to drive the inner bar. In such a case the pattern speeds of the two bars, although unequal, are not independent, so that the regions of two primary resonances of the two bars overlap. Such cases have been well studied theoretically (Tagger et al. 1987, Sygnet et al. 1988), and many numerical simulations showed examples where the ILR of the outer bar coincides with corotation of the inner one (e.g. FM).

Since both observations and simulations show that the existence of an inner bar is a distinct possibility, G. D. van Albada and I made a large number of simulations of the gas response to such a two-bar system. This work, still in progress, as well as the work of FM, shows clearly that the existence of an inner bar increases substantially the inflow in the inner 
regions compared to single bar systems. We are now focusing on the effects the different parameters of the inner bar have on the amount of inflow.

\subsection{CONCLUSIONS}

1) Gas flows in the inner regions of barred galaxies with shocks imply important inflow, which is highest in the region of the shock.

2) The inflow is high during or after violent events like the formation of bars, and much less during their quasi-steady evolution.

3) The rate of inflow is higher for thinner, slower rotating bars in more centrally concentrated galaxies. The same is true if the recycling or the viscosity is higher.

4) The main results have been found by more than one method of modeling the interstellar medium, although quantitative differences do exist.

5) Bars can push gas further in if there is no ILR, but there are indications that ILRs exist in at least a fair fraction of barred galaxies.

6) A secondary bar enhances the inflow in the central regions and leads the gas nearer to the center.

\section{References}

Athanassoula E. (1992) Mon. Not R. Astr. Soc., 259, 345. (A92b)

Athanassoula E. (1994) Mass-Transfer Induced activity in Galaxies, ed. I. Shlosman, Cambridge University press, p. 143. (A94)

Combes F. (1994) Mass-Transfer Induced activity in Galaxies, ed. I. Shlosman, Cambridge University press, p. 170. (A94) 233, 82.

De Vaucouleurs G. (1974) The formation and Dynamics of Galaxies, ed. J.R. Shakeshaft, Dordrecht, Reidel, p.1.

De Vaucouleurs G. (1975) Ap. J. Sup., 29, 193.

Devereux N. A., Kenney J. D. P. and Young J.S. (1992) A. J., 103, 784.

Friedli D. and Benz W. (1993) Astr. Ap., 268, 65. (FB)

Friedli D. and Martinet L. (1993) Astr. Ap., 277, 27. (FM)

Gingold R. A., Monaghan J. J. (1977) Mon. Not. R. Astr. Soc., 181, 375.

Heller C. H. and Shlosman I. (1993) Ap. J. , 424, 84.

Little B. and Carlberg R.G. (1991) Mon. Not. R. Astr. Soc., 250, 161.

Lucy L. B. (1977) $A$. J., 82, 1013.

Schwarz M.P. (1981) Ap. J., 247, 77.

Schwarz M.P. (1984) Mon. Not. R. Astr. Soc., 209, 93.

Shaw M.A., Combes F., Axon D.J. and Wright G.S. (1993) Astr. Ap., 273, 31.

Shlosman I., Frank J. and Begelman M.C. (1989) Nature, 338, 45.

Sygnet J. F., Tagger M., Athanassoula E. and Pellat R. (1988) Mon. Not. R. Astr. Soc., $232,733$.

Tagger M., Sygnet J. F., Athanassoula E. and Pellat R. (1987) Ap. J., 318, L43.

Van Albada G.D., Van Leer B., Roberts W.W. (1982) Astr. Ap., 108, 76.

Wada K. and Habe A. (1992) Mon. Not. R. Astr. Soc., 258, 82. 